Document downloaded from:

http://hdl.handle.net/10251/176220

This paper must be cited as:

Cobollo, C.; Guirao Sánchez, AJ.; Montesinos Santalucia, V. (2020). A remark on totally smooth renormings. Revista de la Real Academia de Ciencias Exactas Físicas y Naturales Serie A Matemáticas. 114(2):1-4. https://doi.org/10.1007/s13398-020-00831-5

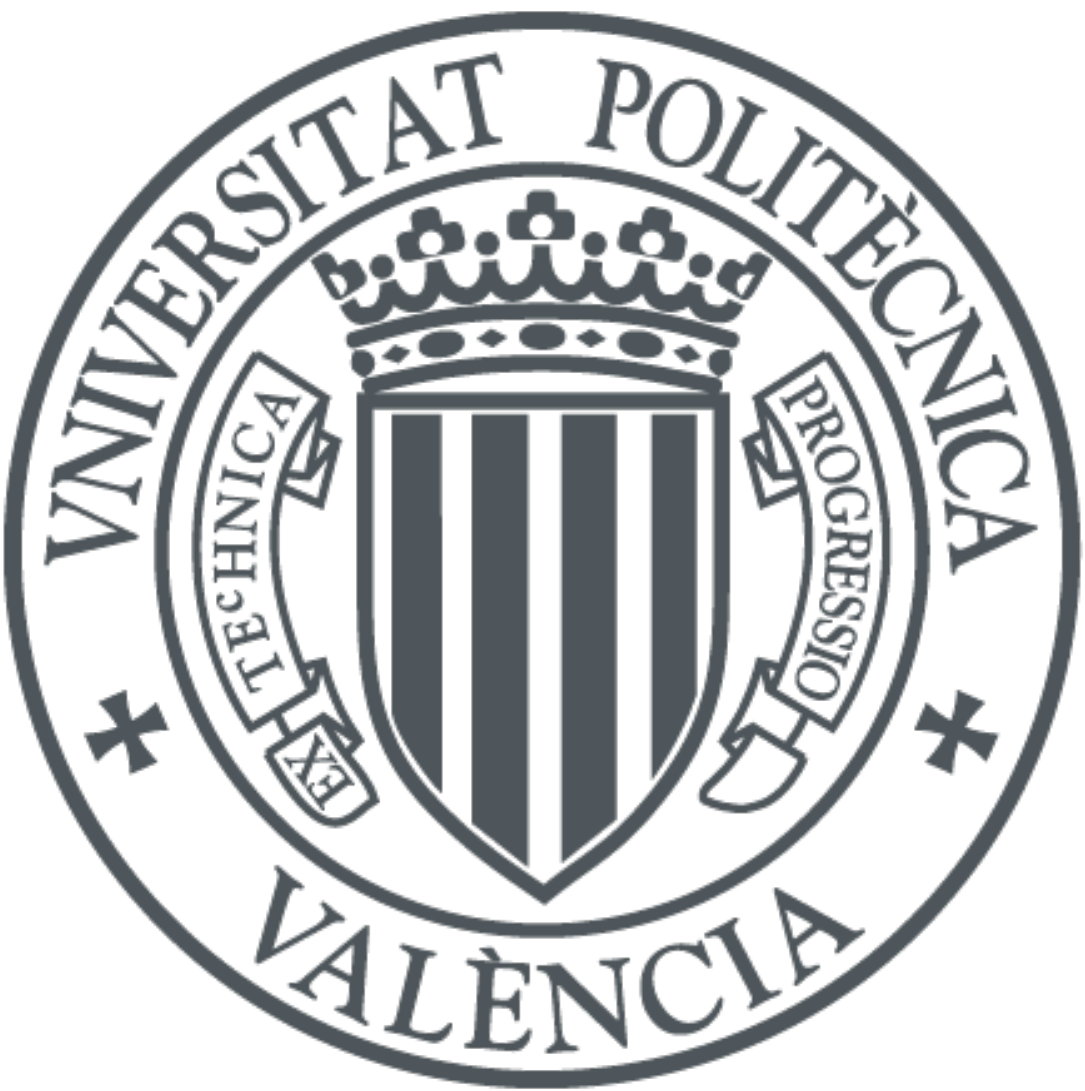

The final publication is available at

https://doi.org/10.1007/s13398-020-00831-5

Copyright Springer-Verlag

Additional Information 


\title{
A remark on totally smooth renormings
}

\author{
Ch. Cobollo • A. J. Guirao • V. \\ Montesinos
}

Received: date / Accepted: date

\begin{abstract}
E. Oja, T. Viil, and D. Werner showed, in Totally smooth renormings, Archiv der Mathematik, 112, 3, (2019), 269-281, that a weakly compactly generated Banach space $(X,\|\cdot\|)$ with the property that every linear functional on $X$ has a unique Hahn-Banach extension to the bidual $X^{* *}$ (the so-called Phelps' property $\mathrm{U}$ in $X^{* *}$, also known as the Hahn-Banach smoothness property) can be renormed to have the stronger property that for every subspace $Y$ of $X$, every linear functional on $Y$ has a unique Hahn-Banach extension to $X^{* *}$ (the so-called total smoothness property of the space). We mention here that this result holds in full generality - without any restriction on the space - and in a stronger form, thanks to a result of M. Raja, On dual locally uniformly rotund norms, Israel Journal of Mathematics 129 (2002), 77-91.
\end{abstract}

Keywords Renormings • Total smoothness · Hahn-Banach smoothness • Local strict convexity

Mathematics Subject Classification (2010) MSC 46B03 - 46B20 . 46B26 - 46B22

Ch. Cobollo

Instituto Universitario de Matemática Pura y Aplicada, Universitat Politècnica de València, Camino de Vera s/n, 46022 Valencia, Spain

E-mail: chrcogo@gmail.com

A.J. Guirao

Instituto Universitario de Matemática Pura y Aplicada, Universitat Politècnica de València, Camino de Vera s/n, 46022 Valencia, Spain

E-mail: anguisa2@mat.upv.es

V. Montesinos

Instituto Universitario de Matemática Pura y Aplicada, Universitat Politècnica de València, Camino de Vera s/n, 46022 Valencia, Spain

E-mail: vmontesinos@mat.upv.es 


\section{A - even more than- totally smooth renorming}

The norm $\|\cdot\|$ of a Banach space $(X,\|\cdot\|)$ is said to be strictly convex (or rotund), if for $x, y \in S_{X}$ such that $\|x+y\|=2$ we have $x=y$. The norm is said to be locally uniformly convex (or locally uniformly rotund) (LUR, for short), if $x \in S_{X}, x_{n} \in S_{X}$ for $n \in \mathbb{N}$, and $\left\|x+x_{n}\right\| \rightarrow 2$ implies $\left\|x_{n}-x\right\| \rightarrow 0$. The norm $\|\cdot\|$ in a dual Banach space is said to have property $w^{*}$-LUR if $x_{n}^{*} \rightarrow x_{0}^{*}$ in the $w^{*}$-topology as soon as $x_{0}^{*}, x_{n}^{*} \in S_{X^{*}}$ for $n \in \mathbb{N}$, and $\left\|x_{n}^{*}+x_{0}^{*}\right\| \rightarrow 2$.

Attention has been paid to the problem of uniqueness of norm-preserving extensions (also called Hahn-Banach extensions) of any continuous functional defined on a closed subspace $Y$ of a Banach space $X$ to the whole of $X$ (a property investigated by R. R. Phelps in [10]). The norm $\|\cdot\|$ of a Banach space $(X,\|\cdot\|)$ has the so-called Hahn-Banach smooth property (HBS, for short) if every $x^{*} \in X^{*}$ has a unique norm-preserving extension to $X^{* *}$. A result that can be traced back to G. Godefroy [5], and that appears also in [7, Lemma III.2.14], says that this property is equivalent to the coincidence of the topologies $w$ and $w^{*}$ on the unit sphere $S_{X^{*}}$ of the dual space $X^{*}$ (we can call this property $\mathbf{W W}^{*}$ Kadets, for short). A stronger property, called total smoothness (TS for short) is that for any closed subspace $Y$ of $X$, any $y^{*} \in Y^{*}$ has a unique Hahn-Banach extension to $X^{* *}$. This is equivalent, by a result of A. E. Taylor and S. R. Foguel [14], [4], to the HBS property plus the rotundness of the dual norm $\|\cdot\|^{*}$. In [13] it was proved that a separable space whose norm has the HBS property has a TS renorming, and in [9] this result was extended to the class of weakly compactly generated spaces (i.e., spaces having a weakly compact linearly dense subset).

Here we just point out that the renorming result holds, even in a stronger form, without any restriction on the space. This observation is based on the following M. Raja's result: If $X^{*}$ is a dual Banach space, then the two following conditions are equivalent: (i) $X^{*}$ admits an equivalent dual LUR norm, and (ii) $X^{*}$ admits an equivalent norm with the $W W^{*}$ Kadets property $[11$, Theorem $3.1]$.

We believe that putting together those results as in Theorem 1 below may help to clarify the connections between the different properties mentioned above.

\{thm-main Theorem 1 Let $(X,\|\cdot\|)$ be a Banach space. Then, the following statements are equivalent:

(i) $X$ has an equivalent norm with property $H B S$.

(ii) $X$ has an equivalent norm whose dual norm has property $W W^{*}$ Kadets.

(iii) $X$ has an equivalent norm whose dual norm is LUR.

(iv) $X$ has an equivalent norm with property $T S$.

Proof $(\mathrm{i}) \Leftrightarrow($ ii) is the aforementioned result of Godefroy [5]. 
(ii) $\Leftrightarrow($ iii) is the quoted result of Raja above [11]. Although not explicitly stated in this reference, it is simple to observe that the topology induced by a dual LUR norm $\|\cdot\|^{*}$ coincides with the $w^{*}$-topology on the unit sphere $S_{X^{*}}$. Indeed, by the LUR property, given $x_{0}^{*} \in S_{X^{*}}$ and $\varepsilon>0$, there exists $\delta>0$ such that if $x^{*} \in S_{X^{*}}$ satisfies $\left\|x_{0}^{*}+x^{*}\right\|>2(1-\delta)$, then $\left\|x_{0}^{*}-x^{*}\right\|<\varepsilon$. Let $\left\{x_{i}^{*}\right\}$ be a net in $S_{X^{*}}$ that $w^{*}$-converges to $x_{0}^{*}$. Find, by Riesz's Lemma, $x_{0} \in S_{X}$ such that $\left\langle x_{0}, x_{0}^{*}\right\rangle>1-\delta$. There exists $i_{0}$ such that $\left\langle x_{0}, x_{i}^{*}\right\rangle>1-\delta$ for $i \geq i_{0}$. Thus, $\left\|x_{0}^{*}+x_{i}^{*}\right\| \geq\left\langle x_{0}, x_{0}^{*}+x_{i}^{*}\right\rangle>2(1-\delta)$ for $i \geq i_{0}$, hence $\left\|x_{0}^{*}-x_{i}^{*}\right\|<\varepsilon$ for $i \geq i_{0}$, and the conclusion follows.

(iii) $\Rightarrow$ (iv) follows from the Taylor-Foguel result [14],[4], quoted above, and the observation in the proof of the equivalence (iii) $\Leftrightarrow($ ii) here.

(iv) $\Rightarrow$ (i) is obvious.

Remark 1 1. Observe that, in particular, the TS norm defined in (iii) above on every Banach space with a HBS norm is Fréchet differentiable.

2. Banach spaces that satisfy one (and then all) of the conditions (i) to (iv) in Theorem 1 have been characterized in other different ways. Let us mention here that, for example, Theorem 1.4 in [3] provides a few of them, in terms of (a) the existence of a dual norm in $X^{*}$ such that $\left(S_{X^{*}}, w^{*}\right)$ is a Moore space, or (b) the existence of an equivalent dual norm such that $\left(S_{X^{*}}, w^{*}\right)$ is symmetrized by a symmetric $\rho$ such that every point $x^{*} \in S_{X^{*}}$ has $w^{*}$-neighborhoods of arbitrary small $\rho$-diameter, or (c) the existence of a dual equivalent norm such that $\left(S_{X^{*}}, w^{*}\right)$ is metrizable, or even (d) that $\left(B_{X^{*}}, w^{*}\right)$ is a descriptive compact space (for details, see the op. cit. and the reference list there).

Remark 2 Let us mention here (only with a hint for the proofs) that, for a Banach space $(X,\|\cdot\|)$ whose norm $\|\cdot\|$ has property HBS,

1. The norm, restricted to any closed subspace of $X$, has property HBS too, a consequence of the $w^{*}$-lower semicontinuity of the dual norm.

2. $X$ is Asplund, as it follows from (i) in Theorem 1 and a separable reduction argument.

3. $X$ is nicely smooth (i.e., there is no proper 1-norming subspace in $X^{*}$ ) and that, in fact, every James boundary is strong (see, e.g., [1, Paragraph 3.11.8.3]).

4. If $(X,\|\cdot\|)=\left(C(K),\|\cdot\|_{\infty}\right)$, where $K$ is a compact topological space, then $K$ is finite. This follows from the fact that the set of extreme points of $B_{C(K)^{*}}$ is $\left\{ \pm \delta_{k}: k \in K\right\}$, that all extreme points are distributed between two closed hyperplanes, the Krein-Milman theorem, and the consequent reflexivity of the space $C(K)$. This observation depends strongly on the fact that the norm on $C(K)$ is the supremum norm. A space $C(K)$, for $K$ an infinite compact space, may admit an equivalent norm $\|\cdot\|$ whose dual is LUR (and so $\|\cdot\|$ has property HBS): Just take $K$ an infinite countable compact space; it is metrizable and scattered (see, e.g., [1, Lemma 14.21]), hence $C(K)$ is Asplund (see, e.g., [1, Theorem 14.25]). Thus, $C(K)^{*}$ is 
separable, and the conclusion follows from a classical result of Kadets (see, e.g., [2, Section 2]).

5. There exists a LUR renorming of $X$. This follows from the aforementioned Raja's result and a result of $\mathrm{R}$. Haydon in [8]. Note that it is an open problem (see, e.g., [12, Problem 1] and [6, Problem 102]) whether a space $X$ has a LUR renorming as soon as it has a norm whose dual norm has property $w^{*}$-LUR.

Acknowledgements Supported by AEI/FEDER (project MTM2017-83262-C2-2-P of Ministerio de Economía y Competitividad), by Fundación Séneca, Región de Murcia (grant 19368/PI/14), and Universitat Politècnica de València (A. J. Guirao)

Supported by AEI/FEDER (project MTM2017-83262-C2-1-P of Ministerio de Economía y Competitividad) and Universitat Politècnica de València (V. Montesinos)

We thank the referees for their work, that neatly improved the original version of this note to its final form.

\section{References}

1. M. Fabian, P. Habala, P. Hájek, V. Montesinos, and V. Zizler, Banach Space Theory: The Basis of Linear and Nonlinear Analysis, Springer, New York, Dordrecht, Heidelberg, London (2011)

2. M. Fabian, V. Montesinos, and V. Zizler, Smoothness in Banach spaces. Selected problems, Rev. R. Acad. Cien. Serie A. Mat. RACSAM, 100 (1-2), 101-125 (2006)

3. S. Ferrari, J. Orihuela, M. Raja, Generalized metric properties of spheres and renorming of Banach spaces. Rev. R. Acad. Cienc. Exactas Fís. Natl. Ser. A Math. RACSAM 113, 2655-2663 (2019)

4. S. R. Foguel, On a theorem by A. E. Taylor, Proc. Amer. Math. Soc. 9, 325 (1958)

5. G. Godefroy, Points de Namioka, espaces normants, applications à la théorie isométrique de la dualité, Israel J. Math. 38, 209-220 (1981)

6. A. J. Guirao, V. Montesinos, and V. Zizler, Open Problems in the Geometry and Analysis of Banach Spaces, Springer International Pub. Switzerland (2016)

7. P. Harmand, D. Werner, and W. Werner, M-ideals in Banach Spaces and Banach Algebras, Lecture Notes in Math. 1547, Springer, Berlin (1993)

8. R. Haydon, Locally uniformly rotund norms in Banach spaces and their duals. J. Funct. Anal. 254, 2023-2039 (2008)

9. E. Oja, T. Viil, and D. Werner, Totally smooth renormings, Archiv der Mathematik, 112, 3, 269-281 (2019)

10. R. R. Phelps, Uniqueness of Hahn-Banach extensions and unique best approximation, Trans. Amer. Math. Soc. 95, 238-255 (1960)

11. M. Raja, On dual locally uniformly rotund norms, Israel Journal of Mathematics $\mathbf{1 2 9}$, 77-91 (2002)

12. R. J. Smith, S. L. Troyanski, Renormings of $C(K)$ spaces. Rev. R. Acad. Cienc. Exactas Fís. Natl. Ser. A Math. RACSAM 104 (2), 375--412 (2010)

13. F. Sullivan, Geometrical properties determined by the higher duals of a Banach space, Illinois J. Math. 21, 315-331 (1977)

14. A. E. Taylor, The extension of linear functionals, Duke Math. J. 5, 538-547 (1939) 